\title{
Caractérisation d'une nouvelle souche du virus du flétrissement de la fève isolée de l'artichaut en France
}

\author{
Auguste MIGLIORI, Gian Luigi RANA*, Pasquale PIAZZOLLA**, Jean-Pierre GOURRET*** \& Luisa \\ RUBINO** \\ I.N.R.A., Station de Pathologie végétale, Centre de Recherches de Rennes, F 35650 Le Rheu \\ * Istituto di Patologia vegetale e forestale, Università della Basilicata, 85100 Potenza (Italie) \\ ** Dipartimento di Patologia vegetale. Universita degli Studi, via G. Amendola 165/A, 70126 Bari (Italie) \\ *** Laboratoire de Biologie cellulaire, UA C.N.R.S. $N^{\circ} 256$, Université de Rennes I, F 35042 Rennes Cedex
}

Mots clés additionnels : $B B W V$, biologie, Capitophorus horni, cytopathologie, sérologie.

A virus isolated from artichoke "Camus de Bretagne" in France has been characterized biologically, physicochemically, serologically and cytopathologically. It was identified as a new strain of broad bean wilt virusFrench Artichoke (BBWV-FA) on the following basis: differential host reaction on Chenopodium amaranticolor; physico-chemical properties in good agreement with those of other strains of BBWV; a serological differentiation index of 2-3 between BBWV-FA and another isolate of BBWV from Italian artichoke "Castellammare" (BBWV-IA) ; a relationship between BBWV-FA and BBWV serotypes I and II ; a very slight relationship between BBWV-FA and Lamium mild mosaic virus; ultrastructural aspects of the viroplasms, which contain granular tubules about $70 \mathrm{~nm}$ in diameter.

Additional key words : $B B W V$, biology, Capitophorus horni, serology, cytopathology.

\section{INTRODUCTION}

Plusieurs virus sont observés chez l'artichaut (RANA \& MARTELLI, 1983). En France, l'artichaut qui est principalement cultivé en Bretagne, accuse un affaiblissement depuis une quinzaine d'années. L'étude entreprise, afin de cerner la cause de cette diminution des rendements, a permis d'établir la présence, la répartition, la fréquence et la nuisibilité des virus chez "Camus de Bretagne" (Migliori et al., 1987). Deux virus sur les 6 observés sont fréquents ; il s'agit d'un potyvirus (Artichoke latent virus $=\mathrm{ALV}$ ) et d'un virus sphérique qui se rapproche du Broad bean wilt virus (BBWV) (MigLIORI et al., 1984), que nous avons étudié ici.

Le BBWV signalé chez l'artichaut par Russo \& RANA (1978) a été mentionné pour la première fois par StUBBS (1947) et décrit par TAYLOR \& STUBBS (1972) et, plus récemment par EDWARDSON \& CHRISTIE (1986) et LISA \& BOCCARDO (à paraître). Certains auteurs comme MATTHEWS (1979), EDWARDSON \& CHRISTIE (1986) classent le BBWV dans le groupe des 
Comovirus, d'autres comme DoEL (1975), LISA et al., 1982, RUSSO et al. (1983) le classent dans un groupe distinct.

La fréquence de l'isolat artichaut BBWV-FA (French artichoke) dans les cultures bretonnes, de même que la présence d'un autre isolat BBWV-IA (Italian artichoke) dans les cultures italiennes (RANA et al., 1987), nous ont conduit à comparer ces 2 virus afin de les situer parmi le BBWV.

Les propriétés biologiques, physico-chimiques, sérologiques et cytopathologiques du BBWV-FA sont comparées à celles des BBWV-IA, BBWV sérotypes I et II (UyEmoto \& Provvidenti, 1974) et au Lamium mild mosaic virus (LMMV), (LISA et al., 1982).

Les résultats de cette étude sont présentés et discutés dans cet article.

\section{MATÉRIEL ET MÉTHODES}

Les souches étudiées dans cette étude sont les suivantes :

- Isolats de l'artichaut, BBWV-FA et BBWV-IA. - BBWV sérotype $I$, isolat de la vigne = BBWV-G (CASTROVILLI et al., 1985). Isolat Nasturtium ringspot virus = NRSV (BOCCARDO \& CONTI, 1973).

- BBWV sérotype II, BBWV-PV 131 de l'American type Collection.

- Isolat Lamium mild mosaic virus = LMMV (LISA et al., 1982).

\section{A. Propriétés biologiques}

\section{Transmission mécanique}

BBWV-FA et BBWV-IA sont multipliés sur Chenopodium quinoa Willd. L'inoculum est obtenu par le broyage des tissus foliaires $(1 \mathrm{~g}+4 \mathrm{ml})$ dans du tampon phosphate $0,01 \mathrm{M}$ pH 7 contenant 0,2 p. 100 de DIECA. L'inoculation est effectuée en présence de $80 \mathrm{mg}$ de charbon végétal et $80 \mathrm{mg}$ de carborundum 600 mesh par $\mathrm{ml}$ d'inoculum sur une gamme d'hôtes différentiels placés en serre à $20-24^{\circ} \mathrm{C}$.

\section{Transmission par pucerons}

Les essais de transmission du BBWV-FA sont effectués par Capitophorus horni, Börner et Myzus persicae, Sulz, selon le mode non persistant. Dans le cas de BBWV-IA, BBWV-G et BBWV-PV 131, seul M. persicae a été utilisé.

Des groupes de 10 pucerons aptères, maintenus en élevage sur artichaut sain ou sur $C$. quinoa, sont déposés après un jeûne de $2 \mathrm{~h}$ sur un plant d'artichaut infecté mécaniquement par les virus cités ci-dessus. Les pucerons observés à la loupe binoculaire sont récupérés 1 à $2 \mathrm{~min}$ après leur prise alimentaire et déposés ( 5 individus par plant) sur 10 artichauts "Camus de Bretagne» et 10 Physalis floridana, Rydb.

\section{B. Propriétés physico-chimiques}

Les caractéristiques d'inactivation et de limite de dilution du BBWV-FA et du BBWV-IA sont détermi- nées in vitro à partir d'extraits $1 \mathrm{~g} / \mathrm{ml}$ obtenus de C. quinoa infecté de façon systémique. Le contrôle est effectué pour chaque cas sur 6 plants de $C$. quinoa.

La technique de purification appliquée pour le BBWV-FA et pour les autres isolats est celle utilisée par LISA (commun. personn.) pour le BBWV isolé de courgette.

Après broyage des feuilles en présence de citrate trisodique $0,25 \mathrm{M}$ et 0,1 p. 100 d'acide thioglycolique, le virus est clarifié, traité au triton $X-100$ (5 p. 100) puis sédimenté $2 \mathrm{~h}$ à $82000 \mathrm{~g}$. Après une seconde clarification suivie d'une sédimentation $(2 \mathrm{~h}$ à $82000 \mathrm{~g})$ le virus est déposé sur des gradients obtenus après décongélation de la solution de citrate trisodique contenant 20 p. 100 de saccharose. L'analyse effectuée après $3 \mathrm{~h}$ de centrifugation à $197000 \mathrm{~g}$ (Rotor SW-41) à l'aide d'un analyseur ISCO 640, permet de séparer les fractions virales. Celles-ci sont sédimentées pendant $3 \mathrm{~h}$ à $146000 \mathrm{~g}$ et reprises dans une solution de citrate trisodique $0,025 \mathrm{M}, \mathrm{pH} \mathrm{7,5}$.

La détermination des coefficients de sédimentation et des densités en équilibre (buoyant densities), des composants viraux est effectuée par ultracentrifugation avec la centrifugeuse Modèle $\mathrm{E}$. Beckman équipée de l'optique SCHLIEREN.

La centrifugation pour l'équilibre en sulfate de césium est effectuée à $25^{\circ} \mathrm{C}$ pendant $16 \mathrm{~h}$ à 44000 r.p.m.

L'acide nucléique est extrait à partir des composants « Middle » et «Bottom» des BBWV-FA et IA, par le phénol-dodésyl-sulfate de sodium (SDS), précipité en présence d'éthanol à $25^{\circ} \mathrm{C}$, puis mis en suspension avec de l'eau distillée stérile. La masse moléculaire est déterminée par électrophorèse en gel d'agarose $1,2 \mathrm{p}$. 100 en tampon tris-borate pendant $1 \mathrm{~h}$ à $100 \mathrm{~V}$. Les échantillons sont dénaturés en présence de 50 p. 100 de formamide déionisée pendant $10 \mathrm{~min}$ à $55^{\circ} \mathrm{C}$. Après migration, le gel est coloré au bromure d'éthidium $(0,5 \mu \mathrm{g} / \mathrm{ml})$. Les ARN ribosomaux de Escherichia coli $\left(1,01\right.$ et $0,53 \times 10^{6}$ daltons) sont utilisés comme marqueurs ainsi que l'ARN du BBWV-PV 131.

La protéine est préparée à partir de particules virales traitées au tris-(hydroxy-méthyl)-aminométhane, $\mathrm{HCl} 0,125 \mathrm{M} \mathrm{pH} 6,8$ en présence 1 p. 100 de 2 mercaptoéthanol à $100^{\circ} \mathrm{C}$ durant 2 à $3 \mathrm{~min}$. Les protéines sont soumises à l'électrophorèse en gel de polyacrylamide à 15 p. 100 selon la technique décrite par LAEMMLI (1970). La masse moléculaire des polypeptides est déterminée en utilisant comme marqueurs : la phosphorylase b (94000), le sérum-albumine bovine $(67000)$, l'inhibiteur de la trypsine du soja $(20000)$ et l' $\alpha$-lactoalbumine (14 400).

\section{Propriétés sérologiques}

Les lapins immunisés par les BBWV-FA et IA ont reçu une $1^{\text {re }}$ injection intramusculaire de $1 \mathrm{mg}$ de virus additionné de $1 \mathrm{ml}$ d'adjuvant incomplet de FREUND, suivie de 2 injections intraveineuses de $1 \mathrm{mg}$ de virus sans adjuvant respectivement 7 et $14 \mathrm{j}$ plus tard. Un rappel est effectué en intraveineuse 15 et $20 \mathrm{j}$ après la dernière injection.

Les relations sérologiques sont déterminées par la méthode de double immunodiffusion en milieu gélosé 
composé de 1 p. 100 d'agarose pour électrophorèse en citrate trisodique $0,25 \mathrm{M} \mathrm{pH}$ 7,5. Les antigènes BBWV-FA et BBWV-IA (isolats artichauts) sont comparés à l'aide des antisérums BBWV-G, BBWV-NRSV (sérotype I), BBWV-PV 131 (sérotype II) et LMMV.

L'immunoélectromicroscopie (IEM) est également appliquée selon le principe de MILNE \& LUISONI (1977) pour étudier la parenté sérologique entre le BBWV-FA et le LMMV. L'antisérum LMMV a été utilisé tel que et dilué au $1 / 10,1 / 20$ et $1 / 40$.

\section{Microscopie électronique}

Les composants du BBWV-FA séparés en gradient de saccharose, sont observés au microscope après coloration à l'acétate d'uranyle 2 p. 100 .

Des fragments de Lathyrus odoratus L., Nicotiana benthamiana Dom. infectés par le BBWV-FA sont fixés au glutaraldéhyde 2,5 p. 100 dans une solution cacodylate $0,2 \mathrm{M} \mathrm{pH} 7,4$, puis post-fixés à $4{ }^{\circ} \mathrm{C}$ au tétraoxyde d'osmium 1 p. 100. Après déshydratation à l'éthanol, suivi d'un passage à l'oxyde de propylène, les fragments sont imprégnés d'un mélange EponAraldite qui est soumis à la polymérisation. Les cou- pes ultrafines sont observées au microscope électronique après coloration à l'acétate d'uranyle et au citrate de plomb.

\section{RÉSULTATS}

\section{A. Propriétés biologiques des BBWV-FA et IA}

\section{Transmission mécanique sur hôtes différentiels}

Les BBWV-FA et IA peuvent être transmis aux hôtes herbacés sans difficulté. Le virus est susceptible d'infecter une gamme variée d'hôtes. Les 2 isolats de l'artichaut induisent des réactions relativement analogues sur l'ensemble de la gamme d'hôtes différentiels (tabl. 1). Chenopodium amaranticolor Coste et Reyn, réagit seulement par des lésions locales chlorotiques aux 2 isolats artichauts et par une réaction locale et systémique aux BBWV-G et PV 131.

L'isolat italien se différencie de l'isolat français par des taches jaunâtres sur artichaut et des lésions locales nécrotiques plus prononcées sur les feuilles primaires de Phaseolus vulgaris L. «Pinto ».

\section{TABLEAU 1}

Réactions symptomatologiques des hôtes herbacés à l'infection par BBWV-FA, IA, G et PV 131 .

Symptomatological responses of herbaceous hosts to infection by BBMW-FA, IA, G and PV 131 .

\begin{tabular}{|c|c|c|c|c|c|c|c|c|}
\hline \multirow[b]{2}{*}{ HÔTES } & \multicolumn{8}{|c|}{ RÉACTIONS } \\
\hline & \multicolumn{2}{|c|}{$\begin{array}{c}\text { BBWV-FA } \\
\text { Locale systémique }\end{array}$} & \multicolumn{2}{|c|}{$\begin{array}{c}\text { BBWV-IA } \\
\text { Locale systémique }\end{array}$} & \multicolumn{2}{|c|}{$\begin{array}{c}\text { BBWV-G } \\
\text { Locale systémique }\end{array}$} & \multicolumn{2}{|c|}{$\begin{array}{l}\text { BBWV-PV } 131 \\
\text { Locale systémique }\end{array}$} \\
\hline Celosia cristatata Kyntze & $\ln$ & Mo & I $n$ & Mo & - & - & - & - \\
\hline Gomphrena globosa L. & ta. $r$ & Mo & ta. $r$ & Mo & ta. $r$ & Mo & ta. $r$ & Mo \\
\hline Chenopodium quinoa Willd. & $1 \mathrm{c}$ & Dist, $\mathrm{n}$ & $1 \mathrm{c}$ & Dist, $\mathrm{n}$ & $1 \mathfrak{c}$ & Dist, $\mathrm{n}$ & $1 \mathfrak{c}$ & Dist, $\mathrm{n}$ \\
\hline C. amaranticolor Coste et Reyn & $1 \mathrm{c}$ & $\mathrm{ni}$ & $1 \mathrm{c}$ & $\mathrm{n} \mathrm{i}$ & $1 \mathrm{c}$ & Dist, $n$ & $1 \mathrm{c}$ & Dist, $\mathrm{n}$ \\
\hline \multicolumn{9}{|l|}{ Composées } \\
\hline $\begin{array}{l}\text { Carduus pychnocephalus L. } \\
\text { Cynara scolymus L. }\end{array}$ & $\ln$ & Lat & $\ln$ & Lat & - & - & - & - \\
\hline «Camus de Bretagne » & Lat & Lat & Lat & $\mathrm{ta} \mathbf{j}$ & $\mathrm{n} \mathrm{i}$ & $\mathrm{n} \mathrm{i}$ & Lat & Lat \\
\hline «Locale di Mola » & Lat & Lat & Lat & $\operatorname{ta~j}$ & - & - & - & - \\
\hline \multicolumn{9}{|l|}{ Cucurbitacées } \\
\hline $\begin{array}{l}\text { Cucurbita pepo Duch. } \\
\text { «Fl Diamant } »\end{array}$ & $\mathrm{n} \mathrm{i}$ & $\mathrm{n} \mathrm{i}$ & $\mathrm{n} \mathrm{i}$ & $\mathrm{ni}$ & $\mathrm{ni}$ & $\mathrm{n} \mathrm{i}$ & $\mathrm{ni}$ & $\mathrm{n} \mathrm{i}$ \\
\hline \multicolumn{9}{|l|}{ Légumineuses } \\
\hline Lathyrus odoratus $\mathbf{L}$. & Lat & Mo & Lat & Mo & Lat & Mo & Lat & Mo \\
\hline Phaseolus vulgaris L. «Pinto" & $1 \mathrm{n}$ & $\mathrm{n} \mathrm{i}$ & $\ln$ & $\mathrm{n} \mathrm{i}$ & $\ln$ & $\mathrm{n} \mathrm{i}$ & $\ln$ & $\mathrm{n} \mathrm{i}$ \\
\hline « La Victoire $»$ & $1 \mathrm{j}$ & $\mathrm{n} \mathrm{i}$ & $\mathrm{l} \mathbf{j}$ & $\mathrm{n} \mathrm{i}$ & $\mathrm{l} \mathrm{j}$ & $\mathrm{n} \mathrm{i}$ & $1 \mathrm{j}$ & $\mathrm{n} \mathrm{i}$ \\
\hline Vicia faba L. «Ascott » & Lat & Mo & Lat & Mo & Lat & Mo & Lat & Mo \\
\hline Vigna radiata $\mathrm{L}$. (Wilczev) & ta. cn & Mo n & ta. cn & Mo n & - & - & - & - \\
\hline V. unguiculata L. (Walp) & $\ln$ & Mo & $\ln$ & Mo & $\ln$ & Mo & $\ln$ & Mo \\
\hline \multicolumn{9}{|l|}{ Solanées } \\
\hline Capsicum annuum L. « Bastidon» & $\mathrm{n} \mathrm{i}$ & $\mathrm{ni}$ & $\mathrm{n} \mathrm{i}$ & $\mathrm{n} \mathrm{i}$ & Lat & Mo & $\mathrm{n} \mathrm{i}$ & $\mathrm{n} \mathrm{i}$ \\
\hline Nicotiana benthamiana Domin. & Lat & Mo & Lat & Mo & Lat & Mo & Lat & Mo \\
\hline Physalis floridana Rydb & Lat & Mo & Lat & Mo & Lat & Mo & Lat & Mo \\
\hline
\end{tabular}

$\mathrm{An}=$ anneaux ringspots $; \mathrm{c}=$ chlorotique chlorotic $;$ Dist $=$ distorsion distortion $; \mathrm{f}$. ch $=$ feuille de chêne etch $; \mathrm{j}=$ jaunâtre yellowish $;$ $1=$ lésions lesions; Lat = latent latent; Mo = mosaïque mosaic; $\mathrm{n}=$ nécrotique necrotic; $\mathrm{n}$ i non infecté not infected ; $\mathbf{r}=$ rougeâtre reddish $;$ ta $=$ taches spots $;-=$ non éprouvé not tested. 
Seul le sérotype I (BBWV-G) infecte et provoque des symptômes sur Capsicum annuum L. «Bastidon " et Nicotiana glutinosa L.

L'artichaut est sensible aux BBWV-IA, FA et PV 131. Ces 2 dernières souches ne provoquent pas des symptômes particuliers. L'inoculation des BBWV-G et NRSV n'a, par contre, pas permis d'infecter les cultivars « Camus de Bretagne » et « Violet de Provence».

\section{Transmission par pucerons}

La transmission du BBWV-FA s'est avérée délicate. Le BBWV-FA est transmis par $C$. horni. La transmission du virus est effectuée de l'artichaut à l'artichaut (6 plantes infectées sur 10) mais aussi de l'artichaut à $P$. floridana (3 plantes infectées sur 10).

Dans nos conditions expérimentales, $M$. persicae ne transmet ni le BBWV-FA ni le BBWV-IA. Par contre, il transmet les isolats BBWV-G et BBWV-PV 131.

\section{B. Propriétés physico-chimiques}

\section{Inactivation. Limite de dilution}

L'inoculation du virus après le traitement thermique indique une dénaturation du virus à partir de 50 $55^{\circ} \mathrm{C}$, elle est totale à $60^{\circ} \mathrm{C}$. Le virus perd son pouvoir infectieux après une conservation in vitro de $6 \mathrm{j}$ à $20^{\circ} \mathrm{C}$ ou après dilution à $10^{-4}$. Le BBWV-FA ne se différencie pas du BBWV-IA.

\section{Purification}

La technique utilisée pour BBWV-FA permet d'obtenir environ $2 \mathrm{mg}$ de virus à partir de $100 \mathrm{~g}$ de feuilles de $C$. quinoa infectées.

Après sédimentation en gradient de saccharose, 3 pics sont distingués après analyse du gradient, correspondant aux composants «Top » $(\mathrm{T})$, « Middle » (M) et «Bottom» $(\mathrm{B})$.

\section{Coefficients de sédimentation}

Les 3 composants du BBWV-FA (T, M, B) sont nettement différenciés après sédimentation par centrifugation analytique, comme le montre le diagramme de SCHLIEREN (fig. 1). Les coefficients de sédimentation du BBWV-FA sont quasi identiques à ceux du BBWV-IA et en accord avec ceux du BBWV-G et BBWV-PV 131 (tabl. 2).

TABLEAU 2

Coefficients de sédimentation des 4 souches $d u B B W V$ en citrate trisodique $0,21 \mathrm{M}$.

Sedimentation coefficients of 4 strains of $B B W V$ $0.21 \mathrm{M}$ sodium citrate.

\begin{tabular}{lccc}
\hline \multicolumn{1}{c}{ Virus } & «Top » & « Middle » & 《 Bottom » \\
\hline BBWV-FA & $40(\mathrm{~S})$ & $63(\mathrm{~S})$ & $80(\mathrm{~S})$ \\
\hline BBWV-IA & $37(\mathrm{~S})$ & $61(\mathrm{~S})$ & $76(\mathrm{~S})$ \\
\hline $\begin{array}{l}\text { BBWV-G } \\
\text { sérotype I }\end{array}$ & $43(\mathrm{~S})$ & $69(\mathrm{~S})$ & $85(\mathrm{~S})$ \\
$\begin{array}{l}\text { BBWV-PV 131 } \\
\text { sérotype II }\end{array}$ & $42(\mathrm{~S})$ & $69(\mathrm{~S})$ & $87(\mathrm{~S})$ \\
\hline \hline
\end{tabular}

\section{Densité de la particule virale en équilibre}

Les densités des composants $\mathbf{M}$ et $\mathbf{B}$ du BBWV-FA établies en sulfate de césium sont respectivement de 1,35 et $1,37 \mathrm{~g} / \mathrm{cm}^{3}$.

\section{Masses moléculaires de l'acide nucléique et de la protéine}

L'étude de l'acide nucléique du BBWV-FA, a permis de mettre en évidence la migration de 2 composants ARN dans le gel. Les masses moléculaires sont de $2,1 \times 10^{6}(\mathrm{ARN}-1)$ et $1,3 \times 10^{6}(\mathrm{ARN}-2)$ analogues à celles du BBWV-IA $\left(2,2 \times 10^{6}\right.$ et $\left.1,4 \times 10^{6}\right)$ et du BBWV-PV $131\left(2,2 \times 10^{6}\right.$ et $\left.1,6 \times 10^{6}\right)$.

La migration des protéines du BBWV-FA forme 2 bandes dans le gel, qui correspondent à 2 polypeptides distincts, dont les masses moléculaires estimées à 44000 et 26000 sont similaires à celles du BBWVPV 131 (44000 et 25000 ).

\section{Propriétés sérologiques}

Les 2 isolats obtenus d'artichaut BBWV-FA et IA sont reliés sérologiquement comme le prouvent les réactions hétérologues observées avec les mêmes antiIA et FA. La comparaison des titres homologues et hétérologues de ces antisérums, montre que les 2 isolats ne sont pas sérologiquement identiques; les valeurs de SDI (VAN REGENMORTEL \& VON WECHMAR, 1970) ont été estimés à 2 et 3.

Cette différence est confirmée par les épreuves de double-immunodiffusion qui font apparaître un éperon au point de raccordement des lignes de précipitation relatives à ces 2 virus (fig. 2).

L'isolat français BBWV-FA est également relié aux autres souches de BBWV appartenant aux 2 sérotypes I et II précédemment décrits; mais dans les mêmes conditions expérimentales, il s'avère que les arcs de précipités obtenus en utilisant des antisérums homologues du sérotype II, sont plus prononcés que ceux obtenus avec l'antisérum correspondant au sérotype I (fig. 3).

Les figures 4,5 et 6 précisent les parentés sérologiques existant entre les 2 isolats BBWV-FA et IA et les sérotypes I et II du BBWV. Cependant, les arcs de précipités obtenus avec les isolats BBWV-FA, BBWVIA, BBWV-G et les antisérums sérotype II (fig. 5) et le sérotype I (fig. 6) forment un éperon sans raccordement, ces réactions permettent de différencier les isolats de l'artichaut des sérotypes I et II.

Dans nos conditions expérimentales, les isolats BBWV-FA et IA n'ont pas réagi en double-immunodiffusion avec le sérum anti-LMMV (fig. 4). Par contre, en immunoélectromicroscopie, nous avons pu obtenir la décoration des particules virales du BBWVFA en utilisant l'antisérum LMMV non dilué (fig. 7).

\section{Microscopie électronique}

1. Observation des particules virales après purification

L'observation des 3 composants du BBWV-FA après coloration négative a révélé des particules iso- 

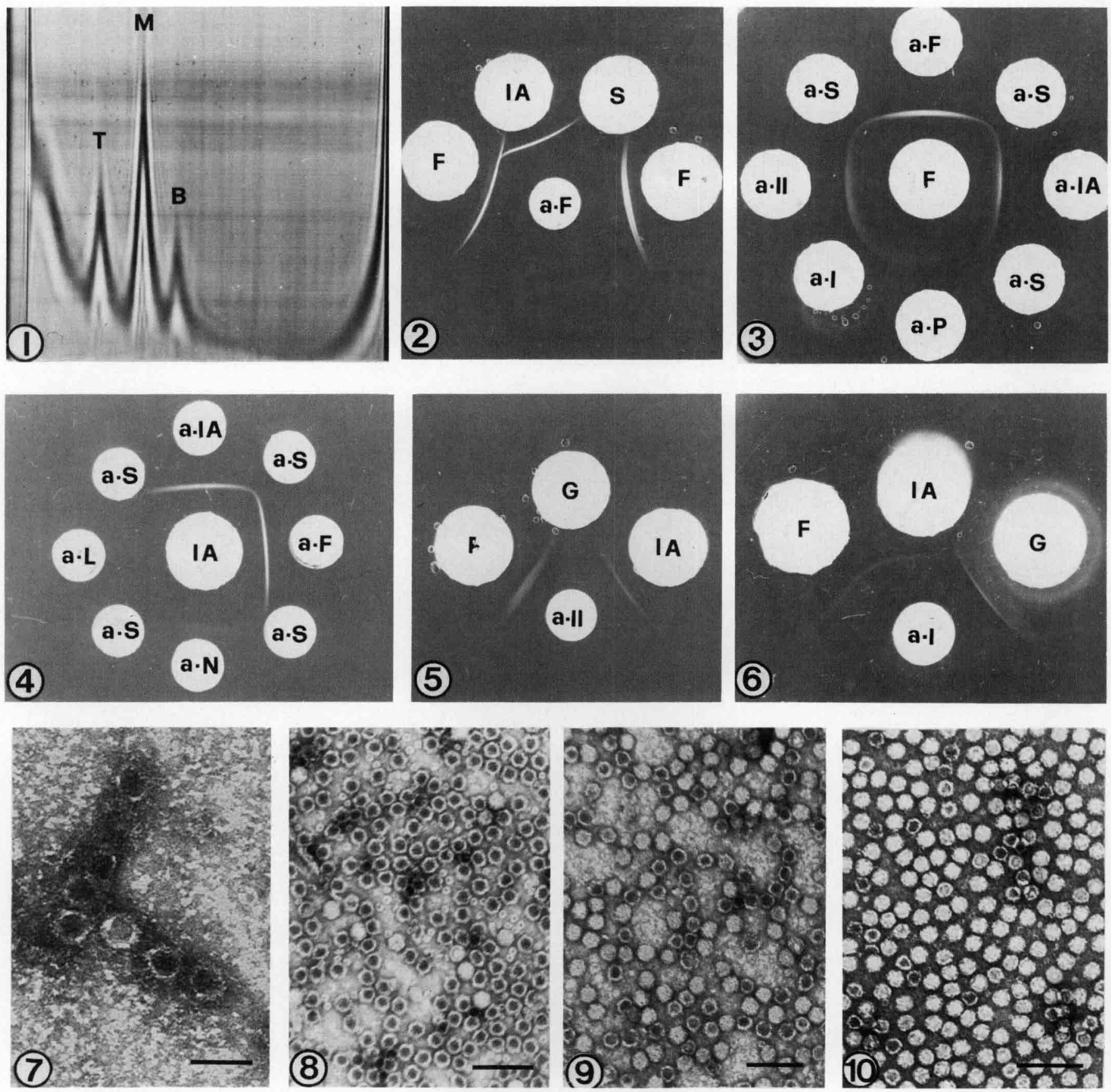

Figure 1

Diagramme Schlieren à la centrifugation analytique d'une préparation virale non fractionnée qui montre 3 composants viraux spécifiques désignés «Top》 (T), «Middle » (M) et « Bottom » (B).

Schlieren diagram of an unfractionated virus preparation in the analytical ultracentrifuge, showing 3 virus-specific components denoted as Top (T), Middle (M) and Bottom (B) sedimenting at different rates.

Figures $2,3,4,5$ et 6

Epreuves sérologiques en double-immunodiffusion en milieu gélosé qui montrent que le $B B W V-F A$ est sérologiquement différent du $B B W V-I A(2)$; les 2 souches artichaut sont sérologiquement reliées au BBWV sérotype II (3 et 5) et au sérotype I (3, 4 et 6).

Double diffusion serological tests in agarose showing that $B B W V$ $F A$ is serologically different from BBWV-IA (2); the two artichoke strains are serologically related to serotype $I I$ of $B B W V(3$ and 5) and serotype I (3, 4 and 6$)$.

Antigènes utilisés à la concentration de $0,5 \mathrm{mg} / \mathrm{ml}$.

Antigens used at a concentration of $0.5 \mathrm{mg} / \mathrm{ml}$.

$F=B B W V-F A ; I A=B B W V-I A ; G=B B W V-G ; S=$ extrait de $\mathrm{C}$. quinoa sain $(S=$ healthy $\mathrm{C}$. quinoa).

Antisérums: $a-F=B B W V-F A ; a-I A=B B W V-I A ; a-I$ $B B W V-I ; a-N=N R S V ; a-I I=B B W V-I I ; a-P=B B W V-P V$ $131 ; a-L=L M M V ; a-S=$ protéines normales de $\mathrm{C}$. quinoa $(a-S=$ normal proteins of $\mathrm{C}$. quinoa).

Figure 7

$B B W V-F A$ décoré par l'antisérum LMMV non dilué, Barre = $50 \mathrm{~nm}$.

$B B W V-F A$ decorated by undiluted $L M M V$ antiserum, Bar = $50 \mathrm{~nm}$.

Figures 8, 9 et 10

Particules virales du BBWV-FA. Composants «Top», les particules sont pénétrées par la coloration négative (8), "Middle " (9) et "Bottom » (10), Barre $=100 \mathrm{~nm}$.

Virus particles of BBWV-FA. Components Top, the particles are penetrated by negative stain (8), Middle (9) and Bottom (10), $B a r=10 \mathrm{~nm}$. 
métriques d'environ 25-27 nm de diamètre. Le composant «Top » est constitué de particules en majorité creuses, pénétrées par le colorant (fig. 8), le
«Middle » est composé de particules entières pour la plupart (fig. 9), le composant «Bottom » est formé de particules entières et bien structurées (fig. 10).
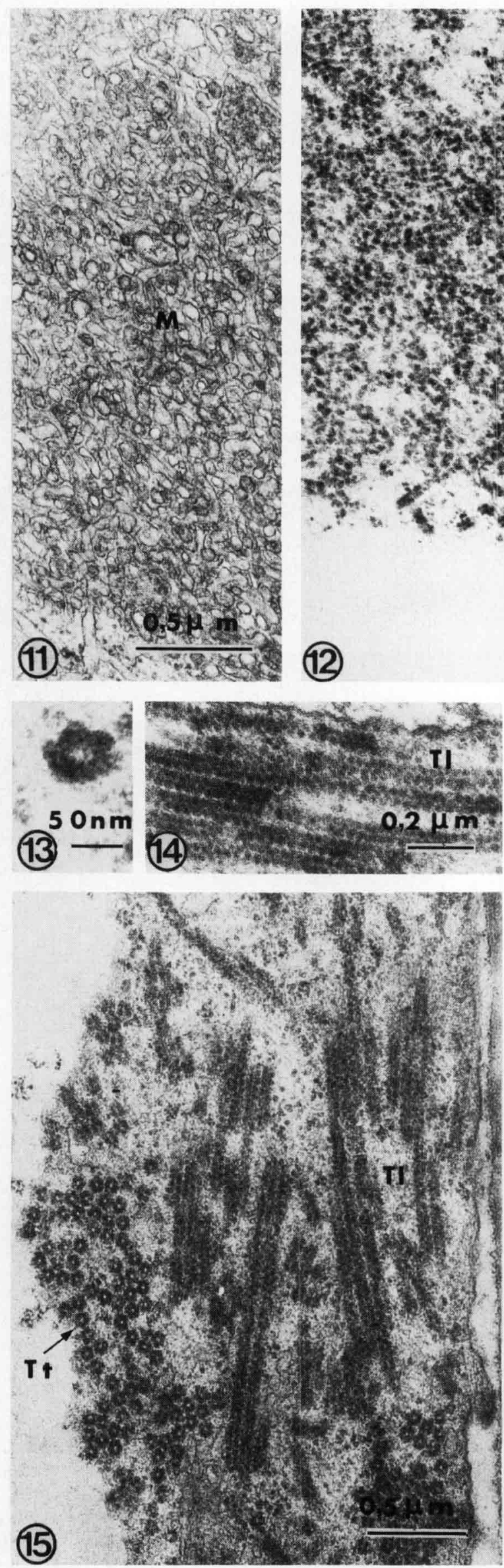
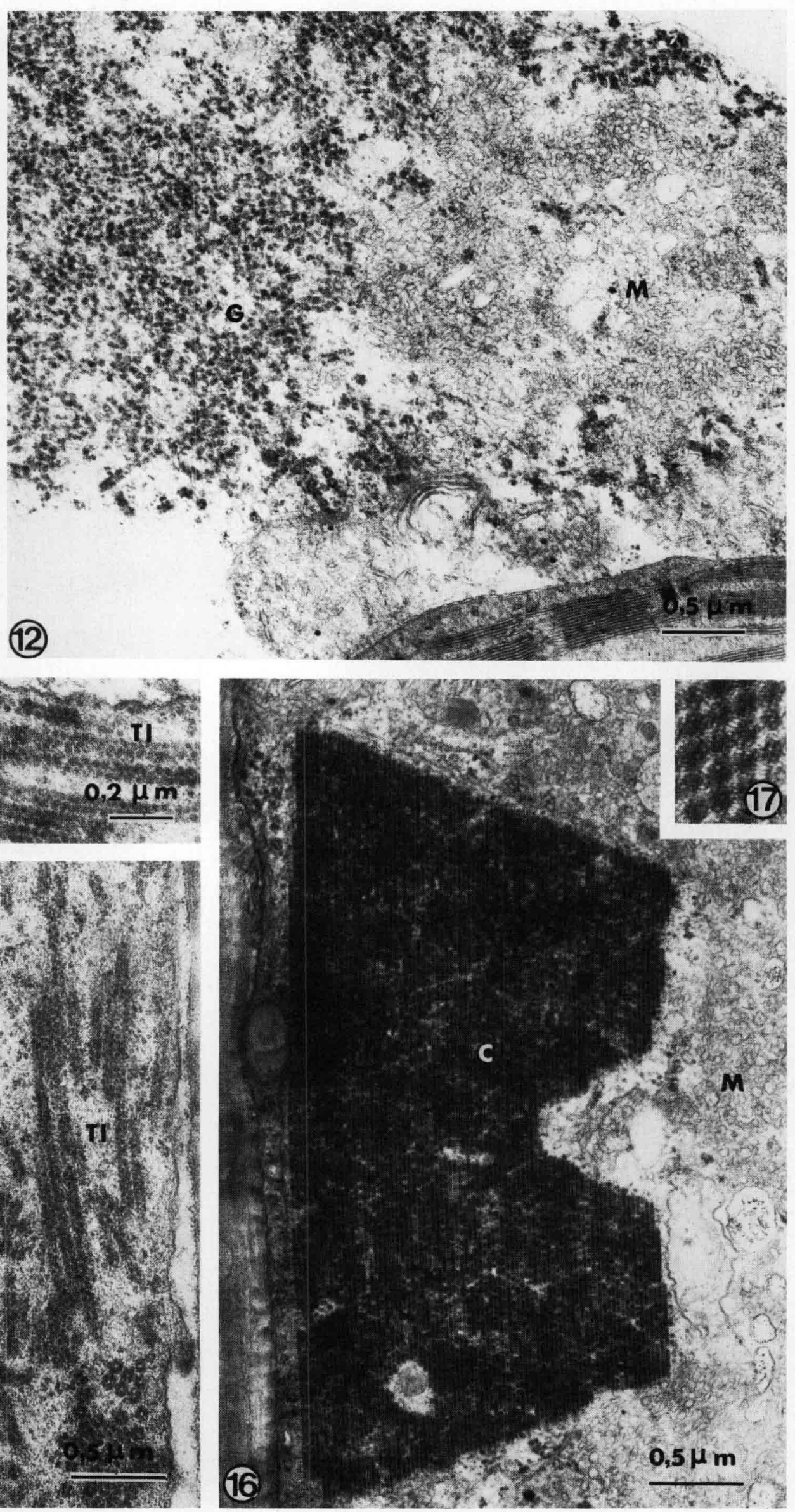


\section{Cytopathologie}

Les cellules de $L$. odoratus et de $N$. benthamiana infectées par le BBWV-FA se distinguent des cellules du témoin par la présence de 1 et parfois 2 viroplasmes plus ou moins volumineux. Ces viroplasmes sont surtout importants dans les cellules du parenchyme chlorophyllien et dans les poils de $N$. benthamiana. Par leur extension au sein du cytoplasme pariétal, ils déforment parfois la vacuole centrale en repoussant le tonoplaste. Les constituants observés dans ces inclusions dépendent du délai écoulé depuis l'inoculation. Les viroplasmes les plus complets s'observent à partir de 10 à $15 \mathrm{j}$. A ce stade, le viroplasme comprend :

a) un réseau membranaire irrégulier de tubules contournés ménageant de place en place des vésicules dilatées à contenu fibreux (fig. 11),

$b$ ) des granules denses à limites peu nettes, mesurant environ 25 à $30 \mathrm{~nm}$ de diamètre dispersés irrégulièrement entre les mailles du réseau membranaire (fig. 12),

c) des tubules granulaires légèrement flexueux, ayant un diamètre d'environ $70 \mathrm{~nm}$ isolés ou regroupés en faisceaux d'orientation variable mais demeurant parallèles au tonoplaste (fig. 14 et 15). Les granules de 25 à $30 \mathrm{~nm}$ formant les tubules sont constitués autour d'un axe creux d'environ $15 \mathrm{~nm}$, cette disposition confère aux sections transversales des tubules un contour pentagonal (fig. 13 et 15),

d) un cristal de granules bien délimités mesurant environ $27 \mathrm{~nm}$ à centre plus ou moins dense aux électrons (fig. 16 et 17 ),

e) quelques globules lipidiques.

Des mitochondries nombreuses et parfois quelques dictyosomes sont observés à la limite du viroplasme.

Les granules des tubules et des cristaux sont assimilés à des virions pouvant, dans certains cas, être formés de la capside vide ne renfermant pas d'ARN. Les granules évoqués en $b$ forment les plages granuleuses irrégulières du viroplasme, représentant probablement des virus immatures. Il est à noter aussi que les tubules granulaires et les cristaux s'excluent mutuellement. Enfin, ni l'un ni l'autre ne s'observent en début d'infection. Le viroplasme est très restreint et ne comporte qu'un réseau membranaire. Celui-ci se développe, acquiert des plages granuleuses et commence à produire soit des tubules de virions, soit des cristaux (fig. 14,15 et 16 ).

\section{DISCUSSION ET CONCLUSION}

Les résultats obtenus dans cette étude, permettent de différencier le BBWV-FA des autres souches connues.

Cette différence est établie au niveau des hôtes différentiels, du vecteur, de la sérologie et de la cytopathologie.

- Hôtes différentiels. Les réactions de quelques hôtes infectés par le BBWV-FA diffèrent de celles obtenues avec les souches connues du BBWV. Le BBWV-FA et le BBWV-IA se différencient surtout chez $C$. amaranticolor par une réaction locale, tandis que $C$. quinoa réagit par des lésions locales et une déformation apicale. Les 2 Chenopodium sont toujours mentionnés comme étant sensibles au BBWV (JURETIČ et al., 1970 ; FROWD \& TOMLINSON, 1972 ; GRACIA \& FELDMAN, 1976 ; WEIDEMANN et al., 1975 ; VEGA et al., 1980; LISA et al., 1982; EDWARDSON \& CHRISTIE, 1986) à l'exception de l'isolat du petit pois observé en Egypte qui induit une réaction locale chez les 2 Chenopodium (KISHTAH et al., 1978). Le BBWV-IA se distingue essentiellement du BBWV-FA par des taches jaunâtres chez Cynara scolymus BBWV-FA et BBWV-PV 131 infectent ce dernier sans induire de symptôme. BBWV-G et NRSV (sérotype I) n'infectent pas $C$. scolymus dans nos conditions expérimentales. Cette observation suppose que le BBWV signalé chez l'artichaut en Italie par Russo \& RANA (1978) pourrait être plus apparenté au sérotype II qu'au sérotype I.

- Vecteur. Le BBWV-FA est transmis par C. horni et non par $M$. persicae, vecteur cité pour toutes les souches étudiées du BBWV (TAYLOR \& STUBBS,
Figure 11

Réseau membranaire d'un viroplasme $\times 36000$

Membranous reticulate part of a viroplasm $\times 36000$.

Figure 12

Viroplasme montrant à la fois un réseau membranaire et une zone granulaire $\times 26000$.

Viroplasm showing both a membranous reticulate net and a granulate area $\times 26000$.

Figure 13

Coupe transversale d'un tubule de virions. Noter la forme approximativement pentagonale $\times 160000$.

Transverse section of a tubule of virions. Notice the approximate pentagonal shape $\times 160000$.

Figure 14

Tubules de virions vus en coupe longitudinale $\times 50000$.

Longitudinally sectioned tubules of virions $\times 50000$.
Figure 15

Tubules de virions en coupes transversales et longitudinales $x$ 30000 .

Transverse and longitudinal sections of the tubular aggregates of virus particles $\times 30000$.

Figure 16

Cristal de virions $\times 30000$.

Crystal of virus particles $\times 30000$.

Figure 17

Alignement de virions dans un cristal $\times 160000$.

Virus particles aligned within a crystal $\times 160000$.

$G$ : zone granuleuse du viroplasme; $M:$ membranes $; T l:$ tubules de virions en coupe longitudinale; $T t:$ tubules de virions en coupe transversale.

$G:$ granulate area of a viroplasm $; M:$ membranes $; T l:$ longitudinal section of tubular aggregates of virus particles ; $T t:$ transverse section of tubular aggregates of virus particles. 
1972 ; BOCCARDO \& CONTI, 1973 ; SAHAMBI et al., 1973 ; MARROU et al., 1976 ; LOCKHART \& BETZOLD, 1982 ; GRACIA \& GUTIERREZ, 1982 ; EDWARDSON \& CHRISTIE, 1986 ; LISA \& BOCCARDO (à paraître). La transmission du BBWV-FA par $C$. horni s'avère néanmoins délicate.

- Les propriétés physico-chimiques du BBWV-FA et IA sont analogues à celles du BBWV décrites par DOEL (1975), EDWARDSON \& CHRISTIE (1986), LISA \& BOCCARDO (à paraître). Les 2 isolats artichaut, peuvent être, en conséquence, assimilés au BBWV.

- Sérologie. L'étude sérologique a permis d'établir la parenté entre les 2 souches du BBWV-FA et IA, isolées d'artichaut et les autres souches du BBWV appartenant aux 2 sérotypes I et II.

En double-immunodiffusion, les arcs de précipités relatifs aux 2 antigènes BBWV-FA et IA se raccordent en formant un éperon ; ces 2 virus appartiennent par conséquent à des sérotypes différents.

Les arcs de précipités obtenus avec les isolats de l'artichaut et les sérotypes I et II forment un éperon sans se raccorder, cette réaction suppose que les BBWV-FA et IA n'appartiennent pas aux 2 sérotypes mentionnés.

L'immunodiffusion n'a pas révélé de réaction entre le BBWV-FA et IA et le sérum anti-LMMV. Toutefois la parenté sérologique entre le BBWV-FA et le LMMV a été mise en évidence par l'immunoélectromicroscopie. Il s'agit d'une relation sérologique très éloignée, ce résultat conforte ceux obtenus par LISA et al. (1982) établissant une parenté sérologique entre le LMMV et le sérotype I du BBWV, mais aucune parenté entre ce virus et le sérotype II.

- Cytopathologie. Les données ultrastructurales concernant les différentes souches du BBWV sont très nombreuses (RUBIO-HUERTOS, 1968 ; JURETIČ et al., 1970 ; MILIČIČ et al., 1974 et 1976 ; CADILHAC \& MARROU, 1976 ; KISHTAH et al., 1978 ; VEGA et al., 1980). Elles montrent à la fois des caractères constants - le réseau membranaire et les zones granulaires mais aussi une grande variabilité ou polymorphisme des arrangements de virions.

Dans le cas du BBWV-FA, les tubules granulaires ne montrent pas d'association avec les vacuoles secondaires comme ceux cités par RUSSO \& MARTELLI (1975) ; VEGA et al. (1980) et surtout le diamètre $(70 \mathrm{~nm})$ et la structure des tubules sont différents de ceux observés avec les souches du BBWV mentionnés par RuBIO-HuERTOS (1968); RUSSO \& MARTELlI (1975) ; VEGA et al. (1980).

Les propriétés biologiques et sérologiques ont permis de distinguer l'isolat français (FA) de l'isolat italien (IA).

Les caractéristiques biologiques, sérologiques et cytopathologiques permettent de distinguer le BBWVFA des souches connues du BBWV.

Reçu le 22 avril 1987. Accepté le 6 janvier 1988.

\section{REMERCIEMENTS}

Nous remercions vivement Raffaele LAFORTEZZA (Dipartimento di Patologia Végétale, Università degli studi, Bari). Mlle Boguals (Laboratoire de Biologie Cellulaire, Université de Rennes I) pour leur contribution aux préparations en microscopie électronique. Les Prof. LISA \& CONTI (Laboratoire de Phytovirologie appliquée C.N.R. Turin) et le Prof. Provvidentl (Dept. of Plant Pathology New York State Agri. Exp. Station, Geneva U.S.A.) pour les souches et les antisérums.

\section{RÉFÉRENCES BIBLIOGRAPHIQUES}

Boccardo G., Conti M., 1973. Purification and properties of Nasturtium ringspot virus. Phytopathol. Z, 78, 14-24.

Cadilhac B., Marrou J., 1976. Virus du flétrissement de la Fève (BBWV) : une étude ultrastructurale. Ann. Phytopathol., 8 (4), 471-476.

Castrovilli, Savino V., Castellano M. A., Engelbrecht D. J., 1985. Characterization of a grapevine isolate of broad bean wilt virus. Phytopath. mediterr., 24, 35-40.

Doel T. R., 1975. Comparative properties of type, nasturtium ringspot and petunia ringspot strain of broad bean wilt virus. J. gen. Virol., 26, 95-108.

Edwardson J. R., Christie R. G., 1986. Viruses infecting forage legumes. Vol. I. Monograph., 14, 95-100.

Frowd J. A., Tomlinson J. A., 1972. Relationship between a parsley virus, nasturtium ringspot virus and broad bean wilt virus. Ann. Appl. Biol., 72, 189-195.

Gracia O., Feldman J. M., 1976. Studies on weed plant as sources of viruses. IV. Broad bean wilt virus in Sisymbrium irio and Plantago lanceolata in Argentine. Phytopathol. Z, 85, 227-236.

Gracia O., Gutierrez L. S., 1982. Broad bean wilt virus in pepper crops in Argentina. Phytopathol. mediterr., 21, 107-109.

Juretix N., Miličič D., Schmelzer, 1970. Zur Kenntnis des Ringmosaik-Virus der Kapuzinerkress (nasturtium ringspot virus) une seiner Zell-Einschlusskörper. Acto Bot. Croat., 29, 17-26.

Kishtah A. A., Russo M., Tolba M. A., Martelli G. P., 1978. A strain of broad bean wilt virus isolated from pea in Egypt. Phytopathol. mediterr., 17, 157-164.
Laemmli U. K., 1970. Cleavage of structural proteins during the assembly of the head of bacteriophage T4. Nature, London., 227, $680-685$.

Lisa V., Luisoni E., Boccardo G., Milne R. G., Lovisolo O., 1982. Lamium mild mosaic virus : a virus distantly related to broad bean wilt. Ann. Appl. Biol, 100, 467-476.

Lisa V., Boccardo G. Broad bean wilt virus and allied viruses. In : HARRISON \& MURANT. The plant viruses. Viruses with bipartite RNA genomes and isometric particles. Plenum, New York (à paraître).

Lockhart B. E. L., Betzold J. A., 1982. Broad bean wilt virus in Begonia in Minnesota. Plant Dis., 66, 72-73.

Marrou, Duteil M., Leclant F., Cadilhac Brigitte, Esvan C., 1976. Mise en évidence du Virus du Flétrissement de la Fève Broad bean wilt virus (BBWV) dans des laitues présentant des symptômes de marbrure. Ann. Phytopathol., 8 (4), 461-469.

Matthews R. E. F., 1979. Classification and nomenclature of viruses. Intervirology, 12 (3-5), 132-296.

Migliori A., Lot H., Pecaut P., Duteil, Rouze-Jouan J., 1984. Les virus de l'artichaut. I. Mise en évidence de 3 virus dans les cultures françaises d'artichaut. Agronomie, 4, 257-268.

Migliori A., Homo E., Corre J., Marzin H., Le Gal V., Curvale J. P., 1987. Répartition, fréquence et nuisibilité des virus chez l'artichaut en Bretagne. P.H.M. Rev. hortic., 274, 29-36.

Miličič D., Wrischer M., Juretič N., 1974. Intracellular inclusion bodies of broad bean wilt virus. Phytopath., Z., 80, 127-135. 
Miličič D., Juretič N., Plese N., Wrischer M., 1976. Some data on cell inclusions and natural hosts of broad bean wilt virus. Acta Bot. Croat., 35, 17-24.

Milne R. G., Luisoni E., 1977. Rapid immune electron microscopy of virus preparations. In: Methods in Virology, 6 (K. MARAMO ROSCH \& H. Koprowski, Eds), Academic Press, New York, 265281.

Rana G. L., Martelli G. P., 1983. Le virosi delle piante ortive : Virosi del carciofo. L'Italia agricola. Gennaio-Marzo, anno 120, 27 38 .

Rana G. L., Migliori A., Ragozzino A., 1987. La maculatura gialla del carciofo in Campania e Puglia. Inf. Fitopatol., 37 (4), $41-43$.

Rubio-Huertos M., 1968. Further studies on ultrastructure of plants infected with Petunia ringspot virus. Protoplasma, 65, 465-476.

Russo M., Martelli G. P., 1975. Light and electron microscopy of broad bean wilt virus intracellular inclusions. J. Submicr. Cytol., 7 , 335-347.

Russo M., Rana G. L., 1978. Occurrence of two legume viruses in artichoke. Phytopathol., mediterr., 17, 212-216.

Russo M., Savino V., Vovlas C., 1983. Virosi della fava. L'Italia agricola. Le virosi delle piante ortive Gennaio-Marzo, anno 120, 83 95 .
Sahambi H. S., Milne R. G., Cook S. M., Gibbs A. J., Woods R. D., 1973. Broad bean wilt and nasturtium ringspot virus are related. Phytopathol. Z., 76, 158-165.

Stubbs L. L., 1947. A destructive vascular wilt virus disease of broad bean (Vicia faba L.) in Victoria. J. Dep. Agric. Vict., 46, 323-332.

Taylor R. H., Stubbs L. L., 1972. Broad bean wilt virus. $C M I / A A B$ Descr. Plant Viruses, 81.

Uyemoto J. K., Provvidenti R., 1974. Isolation and identification of two serotypes of broad bean wilt virus. Phytopathology, 64, 1547 1548 .

Vega J., Gracia O., Feldman J. M., 1980. Characteristics of Argentine broad bean wilt virus : cytopathological aspects. Phytopathol. Z., 99, 242-280.

Van Regenmortel M. H. V., Von Wechmar M. B., 1970. A reexamination of the serological relationship between tobacco mosaic virus and cucumber mosaic virus 4. Virology, 41, 330-338.

Weidemann H. L., Lesemann D., Paul H. L., Koening R., 1975. Das broad bean wilt, virus als Ursache für eine neue Vergilbungskrankheit des Spinats in Deutschland. Phytopathol. Z., 84, 215-221. 